\title{
The beamformer and correlator for the Large European Array for Pulsars
}

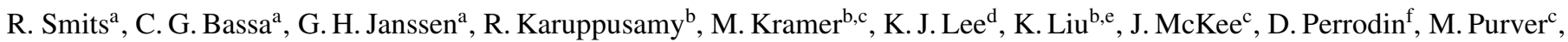 \\ S. Sanidas ${ }^{\mathrm{g}, \mathrm{a}}$, B. W. Stappers ${ }^{\mathrm{c}}$, W. W. Zhu ${ }^{\mathrm{b}}$ \\ aASTRON, the Netherlands Institute for Radio Astronomy, Postbus 2, 7990 AA, Dwingeloo, The Netherlands \\ ${ }^{b}$ Max Planck Institut für Radioastronomie, Auf dem Hügel 69, 53121 Bonn, Germany \\ ${ }^{c}$ Jodrell Bank Centre for Astrophysics, School of Physics and Astronomy, The University of Manchester, Manchester, M13 9PL, United Kingdom \\ ${ }^{d}$ KIAA, Peking University, Beijing 100871, P.R. China \\ ${ }^{e}$ Station de Radioastronomie de Nançay, Observatoire de Paris, 18330 Nançay, France

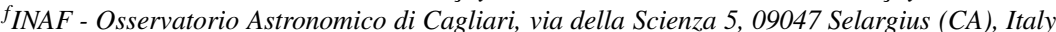 \\ ${ }^{g}$ Anton Pannekoek Institute for Astronomy, University of Amsterdam, Science Park 904, 1098 XH Amsterdam, The Netherlands
}

\section{Abstract}

The Large European Array for Pulsars combines Europe's largest radio telescopes to form a tied-array telescope that provides high 'signal-to-noise observations of millisecond pulsars (MSPs) with the objective to increase the sensitivity of detecting low-frequency gravitational waves. As part of this endeavor we have developed a software correlator and beamformer which enables the formation of a tied-array beam from the raw voltages from each of telescopes. We explain the concepts and techniques involved in the process of adding the raw voltages coherently. We further present the software processing pipeline that is specifically designed to deal with 'data from widely spaced, inhomogeneous radio telescopes and describe the steps involved in preparing, correlating and creating the tied-array beam. This includes polarization calibration, bandpass correction, frequency dependent phase correction, interference mitigation and pulsar gating. A link is provided where the software can be obtained.

Keywords: gravitational waves; techniques: interferometric; pulsars: general

\section{Introduction}

One of the remarkable predictions from the theory of general relativity is the existence of ripples in space-time, called gravitational waves (GWs), which are created for example by the acceleration of masses. The first proof of their existence 'came from the observed decay of the orbital period in compact systems of two orbiting stars as the GWs carry energy away '(e.g. Taylor \& Weisberg, 1982). More recently, the LIGO Scientific Collaboration and the Virgo Collaboration observed the first direct detection of a transient GW signal originating from a binary black hole merger (Abbott et al., 2016). LIGO and 'similar detectors probe GWs at kHz-frequencies making them most sensitive to signals from merging binary neutron stars and black hole systems. Regular observations of radio pulsars have the potential to probe GW frequencies down to nanohertz. This could provide a direct detection of the stochastic GW background originating from the ensemble of coalescing supermassive black hole binaries throughout universe (e.g. Haehnelt, 1994; Jaffe \& Backer, 2003; Sesana et al., 2008) and possibly also a detection of single sources such as near coalescing binary systems and cosmic strings (e.g. Lee et al., 2011; Sanidas et al., 2012; Lommen, 2012). Radio pulsars are spinning neutron stars that emit narrow beams of radio emission along their magnetic axes. As the beam passes across the telescope, a pulse of radiation can be observed with the time between pulses corresponding to the highly regular rotation of the neutron star. This makes

Email address: rjm.smits@gmail.com (R. Smits) pulsars act like cosmic clocks. In a Pulsar Timing Array (PTA) experiment the emission from the most stable pulsars, millisecond pulsars (MSPs), act as the arms of a huge Galactic GW detector (Detweiler, 1979; Hellings \& Downs, 1983). The timing of pulsars for PTAs now spans well over a decade and are typically performed on a monthly basis (e.g. Desvignes et al., 2016; Manchester et al., 2013; The NANOGrav Collaboration et al., 2015). This long time span is what makes a PTA sensitive to GW frequencies down to nanohertz and makes it complementary to the ground-based detectors.

The ongoing efforts for the detection of GWs via a PTA experiment are pushing the precision limits of what is possible when using the existing telescopes individually. They currently fall short in achieving the required timing precision for a large enough sample of pulsars to get a detection (Demorest et al., 2013; Shannon et al., 2015; Lentati et al., 2015). The precision can be improved by increasing the telescope sensitivity.

The Large European Array for Pulsars (LEAP) is an ERCfunded experiment to combine the raw voltages from individual telescopes to form a tied-array telescope to provide high signalto-noise observations of MSPs with the objective to increase the sensitivity for a GW detection (Bassa et al., 2016). It combines the data from the telescopes participating in the European Pulsar Timing Array (EPTA). These telescopes are the Effelsberg telescope (EB), the Lovell telescope at Jodrell Bank (JB), the Nançay radio telescope (NRT), the Sardinia Radio Telescope (SRT) and the Westerbork Synthesis Radio Telescope (WSRT). Combined coherently, the effective area is equivalent to that of 
a 195-m dish.

The observations are performed at L-band from 1332 $1460 \mathrm{MHz}$ in monthly observing sessions of 25 hours. Pulsars are typically observed for 45 minutes to 1 hour. Each pulsar observation is either preceded or followed (or both) by an observation of a phase calibrator for a few minutes. The calibrators were selected from the VLBA Calibrator Lis 1 and are offset by no more than about $5^{\circ}$ from the pulsar position. The Nyquist sampled timeseries from all observations are recorded to disk. These disks are shipped to Jodrell Bank Observatory (JBO) where the baseband data is correlated which yields the exact time-delays and phase-offsets between each pair of telescopes. These delays and offsets are then applied to the timeseries and the timeseries are added together resulting in the LEAP tiedarray beam.

There are several software correlators already in existence. Most notably and versatile are Distributed FX (DiFX) (Deller et al., 2007) and SFXC (Keimpema et al., 2015). DiFX is a correlator for Very Long Baseline Interferometry (VLBI) that can utilize a multi-processor computing environment by parallelizing the computations. It is widely used by radio interferometers. It allows for pulsar binning, but does not do beamforming. SFXC is the EVN data processor and is used to perform global VLBI in real-time. It can perform pulsar binning and both coherent and incoherent dedispersion. In recent years, the ability to phase up the VLBI telescopes to create a tied-array beam has been added. The benefits of developing an independent LEAP correlator and beamformer are the natural integration of the data format used (i.e. the DADA format, van Straten et al., 2012), bandwidths and available computing hardware (see Section 3), the requirement of accurate polarization calibration (see Section 4.2), but also the lack of beamforming capabilities in the existing software correlators at the start of the LEAP project.

Kramer \& Stappers (2010) first introduced LEAP. Bassa et al. (2016) provide a detailed description of its experimental design and initial science results. In this paper we describe in detail the techniques used in the correlation process and the pipeline that has been developed specifically for the purpose of processing the raw voltages from the LEAP telescopes and creating the tied-array beam. All software that has been produced as part of this pipeline is available for download from http://www.epta.eu.org/aom.html.

\section{Technique}

During an observing session, each of the LEAP telescopes observes the same sources simultaneously. The raw voltages are Nyquist-sampled and recorded as baseband data, thereby preserving both the amplitude and phase information of the observed radio waves. This allows the individual time series to be shifted in time and phase offline such that all time series are perfectly aligned as though they were observed with a (very large) single dish. Adding the time series after they have been aligned

1http://www.vlba.nrao.edu/astro/calib in time and phase is called beamforming. The resulting beam is called a tied-array beam.

The time and phase delays between the time series from individual telescopes consists of four components. First, the largest delays are due to differences in the path lengths that the signal has to travel to reach each telescope, called the geometric delay, which corresponds to a time delay. Second, there are differences between each observatory's local clocks which introduces a time delay. The third component consists of instrumentspecific delays due to cables and electronic components which introduce both a time and a phase delay. Finally, the atmosphere (both ionosphere and troposphere) introduces a time delay as well as a phase shift of the radio-wavefront, which depend on the time-varying conditions of the local atmosphere as well as the wavelengths of the radio waves.

The geometric delays can be largely corrected for by using the known terrestrial positions of the telescopes, telescope pointing models and celestial position of the source (calibrator or pulsar). This is achieved using the software program CALC2. The time-varying geometric delays from CALC are stored as polynomials in CALC-files, which are read by the LEAP correlator (see Section 4). For LEAP we make use of a wrapper for CALC, which is part of the software correlator DiFX (Deller et al., 2007). Where known, the pulsar positions are corrected for proper motion to the epoch of the observation. EB is used as the geometric location of the LEAP tied-array beam, even when EB does not participate in the observation session. This fixed reference point is necessary to obtain a consistent LEAP dataset for pulsar timing. The clock delays between the observatories are determined from the local Global Positioning System (GPS) measurements at each observatory. These are performed multiple times per day. For each month the daily average of these measurements are placed on an ftpserver in GPS-files. As part of the LEAP pipeline, these GPSfiles are downloaded for each epoch and read by the LEAP correlator.

The delays from the signal-path and the atmosphere are measured by first cross-correlating the complex sampled voltages of each polarization and each pair of telescopes from either a calibrator or the pulsar signal itself. A cross-correlation is performed by multiplying the Fourier transformed voltage series and integrating over time. This procedure is visualized in Fig. 1. The resulting time-averaged visibilities contain the phase-relationship between the two time series. This can be visualised by plotting the phase of the visibilities as a function of frequency channel as shown in Fig. 2. If they are perfectly in phase, then the resulting visibilities all have a phase of zero. If the phase has a constant value, then the two time series have a phase offset, but no time offset. A time offset between the two time series corresponds to a slope. These can be measured by applying the fringe-find method from Schwab \& Cotton, 1983). This method makes use of phase closure and involves a

\footnotetext{
${ }^{2}$ CALC is part of the Mark-5 VLBI Analysis Software Calc/Solve Ryan \& Vandenberg (1980). The LEAP pipeline uses CALC

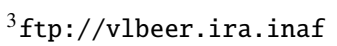
9 


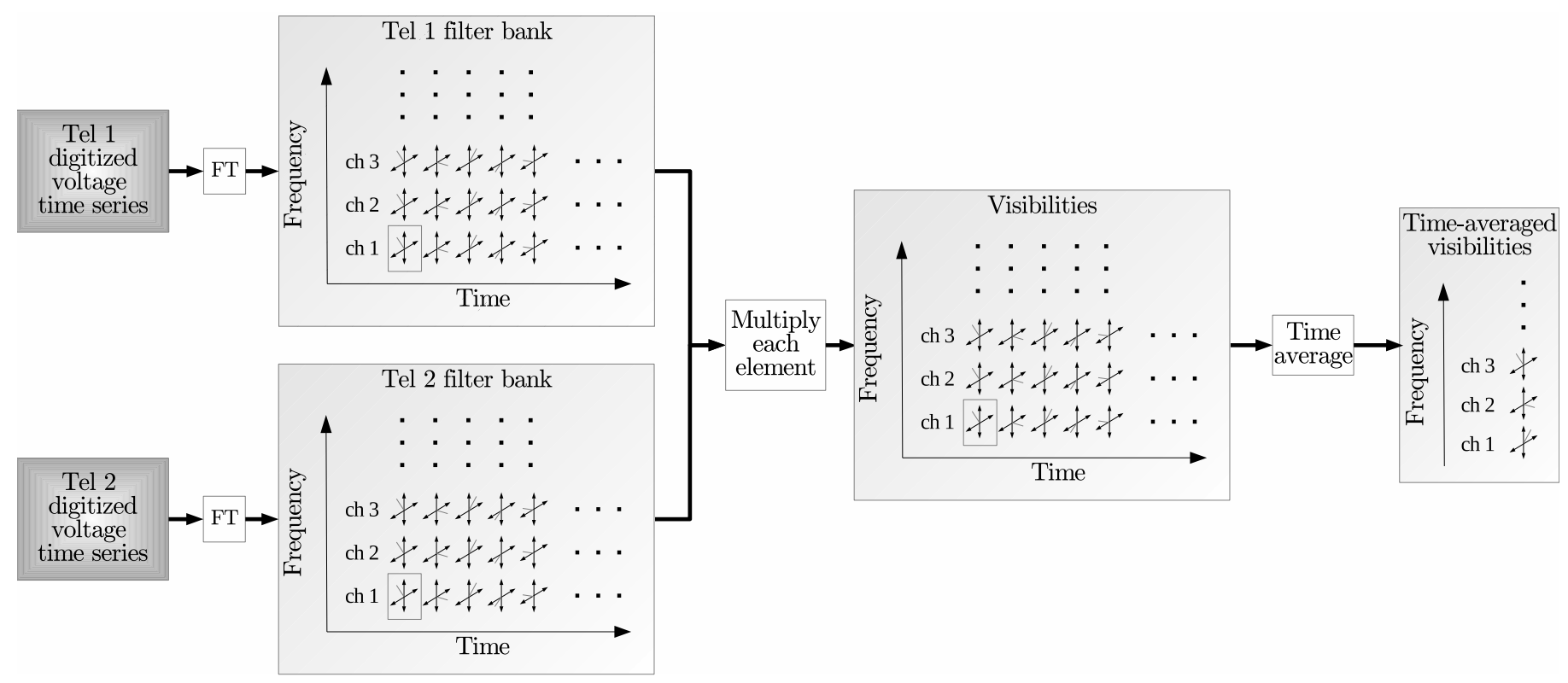

Figure 1: A flowchart of the correlation process. The digitized voltage time series from two telescopes are Fourier transformed to form complex frequency channels, indicated as Tel 1 filterbank and Tel 2 filterbank. Each element is a complex voltage that can be represented as a two-dimensional vector. The corresponding elements from both telescopes are multiplied together to form the visibilities. Each frequency channel is then averaged in time to form the time-averaged visibilities. This process is repeated for all telescope-pairs.

fit of the averaged visibilities to find the fringe solution, which consists of the best values for the time offsets (fringe delay), the phase offsets (fringe phase) and the phase drift (fringe rate) (see Section 4.7.

Once the fringe solution is found, it is applied to the complex samples from all telescopes which aligns them in time and phase. This is done by rotating each complex sample by an amount $\phi[p, t]$, which consists of:

$$
\phi[p, t]=\phi_{\text {samples }}^{p}+\phi_{\text {fringe_delay }}^{p}+\phi_{\text {fringe_offset }}^{p}+\phi_{\text {fringe_rate }}^{p}(t),(1)
$$

where $\mathrm{p}$ is the telescope index and $\mathrm{t}$ is time in seconds. $\phi_{\text {samples }}^{p}$ corrects for the shift of the phases due to the skipped samples as follows:

$$
\phi_{\text {samples }}^{p}=2 \pi \mathrm{N}_{\mathrm{samp}}^{p} \frac{f_{\mathrm{sky}}}{\mathrm{B}},
$$

where $\mathrm{N}_{\text {samp }}^{p}$ are the number of skipped samples for telescope $p$, $f_{\text {sky }}$ is the sky frequency of the band 4 and $\mathrm{B}$ is the bandwidth. $\phi_{\text {fringe_delay }}^{p}$ is the phase from the fractional delay for telescope $\mathrm{p}$. Rotating the complex voltages by this phase corresponds to applying the residual time-delay that is less than one sample. This time-delay consists of the fractional sample delay and the delay measured from the visibilities, which consists of any residual from the predicted clock offset and the contribution from the time-varying ionosphere. The phase from the fractional delay is calculated as follows:

$$
\phi_{\text {fringe_delay }}^{p}=2 \pi \tau_{\text {frac }}^{p} \frac{f_{\text {band }}}{\mathrm{B}},
$$

\footnotetext{
${ }^{4}$ For real sampled data this is the lower edge of the band. For complex sampled data this is the centre frequency.
}

where $\tau_{\text {frac }}^{p}$ is the fractional delay for telescope $\mathrm{p}, f_{\mathrm{band}}$ is the frequency within the downsampled band (from 0 to B). $\phi_{\text {fringe_offset }}^{p}$ is the phase offset of the time-series from telescope $p$ with respect to the time-series from the reference telescope at frequency 0 . This phase offset is calculated from the fringesolution (see Section 4.7) by interpolating the slope of the fringe to a frequency of $0 . \phi_{\text {fringe_rate }}^{p}$ is defined as:

$$
\phi_{\text {fringe_rate }}^{p}(t)=t \cdot r_{\text {fringe }}^{p},
$$

where $t$ is the time in seconds from the start of the fringesolution and $r_{\text {fringe }}^{p}$ is the fringe rate in radians per second for telescope $\mathrm{p}$, which is also a direct product from the fringefitting.

\section{Storage, transport and processing hardware}

The baseband data from every monthly 25-hour session are initially stored at each observatory locally (the pulsar hardware at EB, JBO, SRT and WSRT are described in Karuppusamy et al., 2008; Karuppusamy, 2011). For EB, JBO, NRT and SRT the data are stored in 8 subbands of $16 \mathrm{MHz}$ with the center frequencies at 1340, 1356, 1372, 1388, 1404, 1420,1436 and $1452 \mathrm{MHz}$ for a total bandwidth of $128 \mathrm{MHz}$. The hardware present at the WSRT generates $8 \times 20 \mathrm{MHz}$ subbands. These subbands are thus arranged to overlap with the 8 subbands from the other observatories. At all observatories both polarizations are recorded at 8 bit resolution in the DADA format, where each file contains 10 seconds of one subband. Table 1 shows all the parameters of the recorded baseband data 

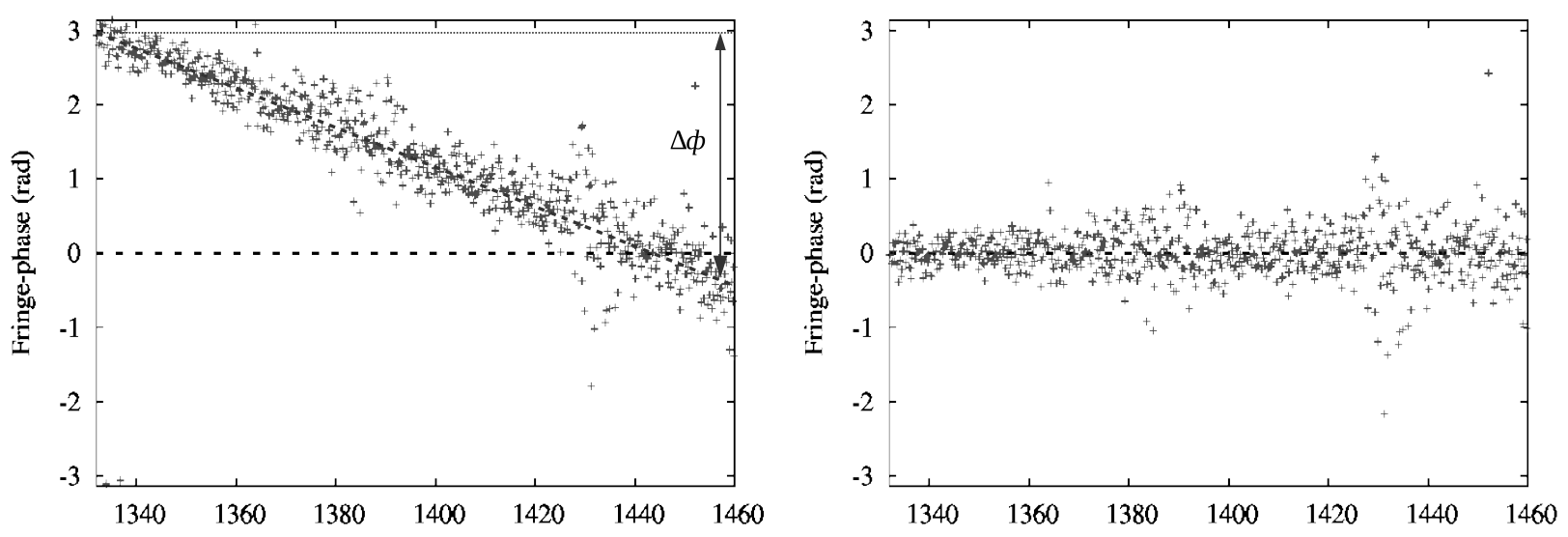

Figure 2: Phase of the visibilities from calibrator J1025+1253 between telescopes EB and NRT as a function of frequency channel (after correcting for the frequency-dependent phase offset, see Section 4.4). The left panel shows a fringe between two telescopes that have a small time offset which introduces a slope. The right panel shows the fringe between two telescopes that are perfectly aligned, both in phase and in time. The scatter in the visibilities is due to the noise in the data, which does not correlate between the telescopes. The increase in scatter around $1430 \mathrm{MHz}$ is due to an artifact in the EB bandpass, as can be seen in the left panel of Fig. 5.

Table 1: The parameters for the baseband data for each of the 5 LEAP observatories as well as for the LEAP data.

\begin{tabular}{llll}
\hline \hline Telescope & $\begin{array}{l}\text { Bandwidth of } \\
\text { subbands }(\mathrm{MHz})\end{array}$ & Polarization & $\begin{array}{l}\text { Nyquist } \\
\text { sampling }\end{array}$ \\
\hline EB & 16 & Circular & complex \\
JB & 16 & Circular & complex \\
NRT & 16 & Linear & complex \\
SRT & 16 & Linear & complex \\
WSRT & 20 & Linear & real \\
LEAP & 16 & Circular & real \\
\hline
\end{tabular}

for each of the observatories, as well as for the resulting LEAP baseband data.

The data volume from one observing session is about $36 \mathrm{~TB}$ for EB, JB and SRT. For NRT it is about $22 \mathrm{~TB}$ because it participates in fewer sources. For WSRT it is about 50 TB due to its larger subbands. These data are stored on 16 times 3 or 4 TB disks.

The baseband data from each site are transported to the JBO either via shipment of the disks, or over the internet for offline processing. The central storage at JBO can hold over two months of unprocessed LEAP data from all the sites, keeping it directly accessible for correlating.

The processing is performed on a high performance computer cluster located at JBO. This cluster consists of 40 nodes, each having two Quad core Intel Xeon processors, 4GB of RAM and 2 TB of storage.

\section{Processing Pipeline}

Once the baseband timeseries from a new observation are available at the central storage, the LEAP pipeline is set up to allow multiple users to process any part of the monthly ob- servations. All files are indexed and their location and relevant header information are stored in a file-location file (FLF), which is made available to all users. The clock correction files are downloaded and are also made available to all users. A polarization calibration procedure is performed on a calibration pulsar which yields a $4 \times 4$ matrix, called a Mueller matrix, for each telescope. From these Mueller matrices, the Jones matrices are produced for each individual observation and each telescope (see Section 4.2). Based on the FLF, a directory structure is created for each user where the FLF is split up into smaller files containing all the relevant information for each individual observation. The CALC files are created for each observation, as well as input files that contain telescope and observation specific information about the correlation or addition. These input files can be modified by software later in the pipeline, or by the user directly.

At this stage the correlations and additions can be performed to form the LEAP baseband time series, containing the tiedarray beam. Creating this time series takes place on the computer cluster and requires several computational steps. Figure 3 shows the flowchart of the LEAP pipeline. There are three sequences, corresponding to an initial calibration of the frequency-dependent phase offsets between all telescope pairs (sequence 1), the correlation of the pulsar signal resulting in the fringe solution (sequence 2) and the application of the fringe solution to the complex samples and formation of the LEAP tiedarray beam (sequence 3). Each sequence consists of copying the required DADA files from the central storage to the computer cluster. The files are read and processed by the LEAP correlator, called lcorr (see Section 4.1).

In sequence 1 the voltages from a calibrator source are polarization calibrated and the geometric delay and clock offsets are applied. The voltages are then correlated, yielding visibilities from each pair of telescopes, which are used to measure the 


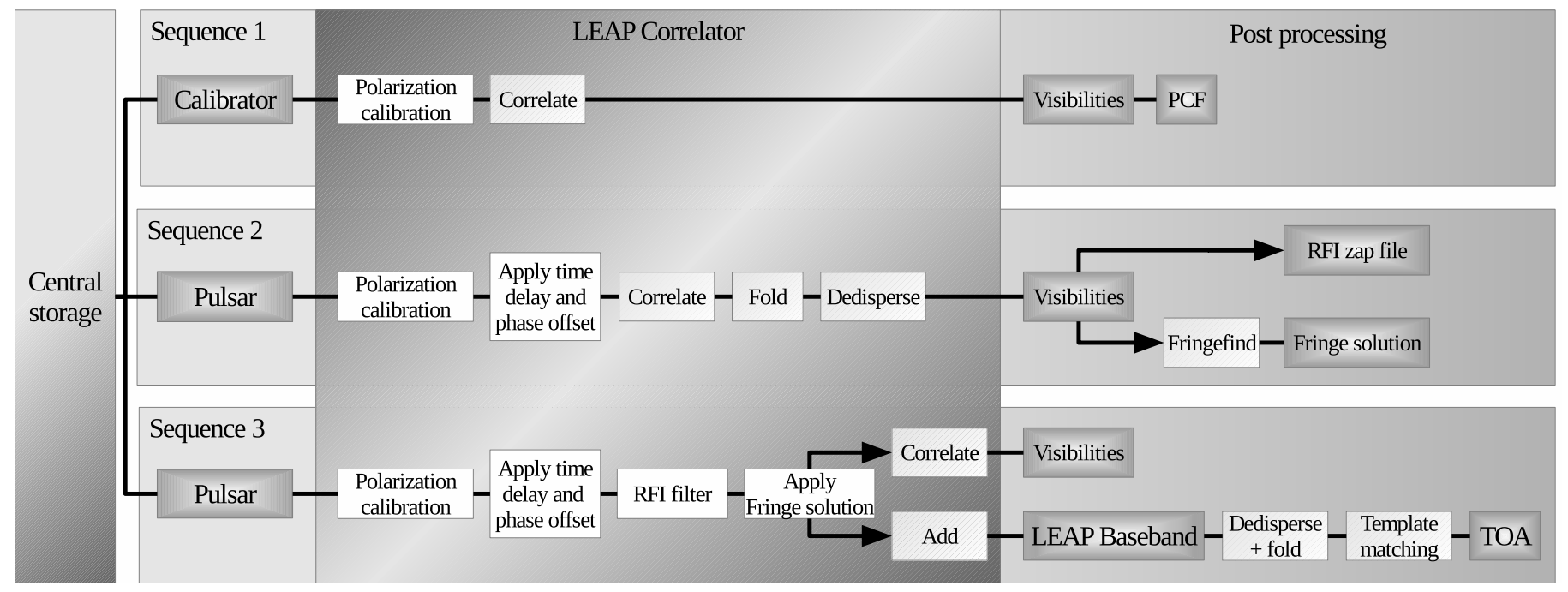

Figure 3: A flowchart of the LEAP pipeline. Each observatory stores the raw voltages from the LEAP observations on disk. The data are then transferred to the central storage (at Jodrell Bank). From the central storage the data is correlated after applying polarization calibration and RFI mitigation filters. The resulting fringe-solutions from each baseline are then applied to the voltages, again after applying polarization calibration and RFI mitigation, and they are added together in phase, resulting in the LEAP phased array. This stage also includes correlating the voltages after the fringe-solution is applied. The resulting visibilities are checked to verify that the applied fringe-solution is indeed correct. The added voltages are processed as normal timing data. They are dedispersed using DSPSR and template matching is performed to produce the final pulse times-of-arrival.

frequency-dependent phase offsets between all telescope pairs (see Section 4.4). These offsets are written to disk in phase correction files (PCFs) and later applied to the raw voltages of the pulsar in sequence 2 and 3 . Sequence 1 only needs to be performed once for the entire observation session.

In sequence 2 the voltages from the pulsar are polarization calibrated and the time and phase offsets from the geometric delays, the clock offsets and the frequency-dependent phase offsets are applied. The voltages are then correlated, folded with the pulsar period and dedispersed to produce the visibilities for each pair of telescopes for each frequency channel and each pulsar phase-bin. The visibilities in the phase-bins with the pulsar signal are averaged together (see Section 4.6) and are then processed via the algorithm as described by Schwab \& Cotton (1983). This yields the phase and time offset and phase drift between the pairs of telescopes, called the fringe-solution (see Section 4.7). Because of clock drifts and the changing ionosphere and troposphere a new fringe-solution needs to be found every five to ten minutes. Thus, this sequence is performed in several independent parts, with each part yielding a new fringesolution. The powerspectrum of the visibilities are searched for outliers, which are marked as radio frequency interference (RFI). Those channels will be 'zapped' when adding the data in sequence 3 .

Sequence 3 is the beamformer. The voltages from the pulsar are again polarization calibrated and the time and phase offsets from the geometric delays, the clock offsets and the frequency-dependent phase offsets are applied. The frequency channels which are marked as RFI are masked by replacing the content with Gaussian noise with mean and rms determined from neighboring time samples (see Section 4.5). The fringe solution is applied. The voltages are then added together, forming the tied array beam, and are written to disk as the LEAP baseband data. The baseband data is dedispersed using DSPSR (van Straten \& Bailes, 2011). Template matching is performed to produce the final pulse times-of-arrival (TOAs) (Taylor, 1992). The LEAP baseband data is stored on a longterm archive. In parallel to adding the voltages, the voltages are also correlated as in sequence 2 , so as to produce the visibilities with the exact same fringe solution as the tied array beam.

All steps are performed by user interaction with code written in $\mathrm{C}$, python and bash scripts.

\subsection{The LEAP correlator}

At the heart of the LEAP correlations lies the C-based program lcorr which reads the individual files containing the digitized voltage time series from each telescope, applies the time and phase corrections, polarization calibration, RFI mitigation and can either correlate the time series to produce the visibilities (mode 1), add them together to produce the tied-array (mode 2), or write out the complex samples for each telescope (mode 3).

Figure 4 shows the flowchart of 1 corr, which describes the 'LEAP Correlator' block in Fig. 3. The program runs on ten seconds of data from each telescope for one frequency band at a time. It first reads the input file which contains the input parameters for the correlation or addition, which includes the phase and time offset for each telescope if these have been obtained from an earlier correlation. lcorr also reads the files containing the Jones matrices, the CALC files, the frequencydependent phase offsets, the bandpass and the GPS clockfiles. The Jones matrices are used for polarization calibration. The 


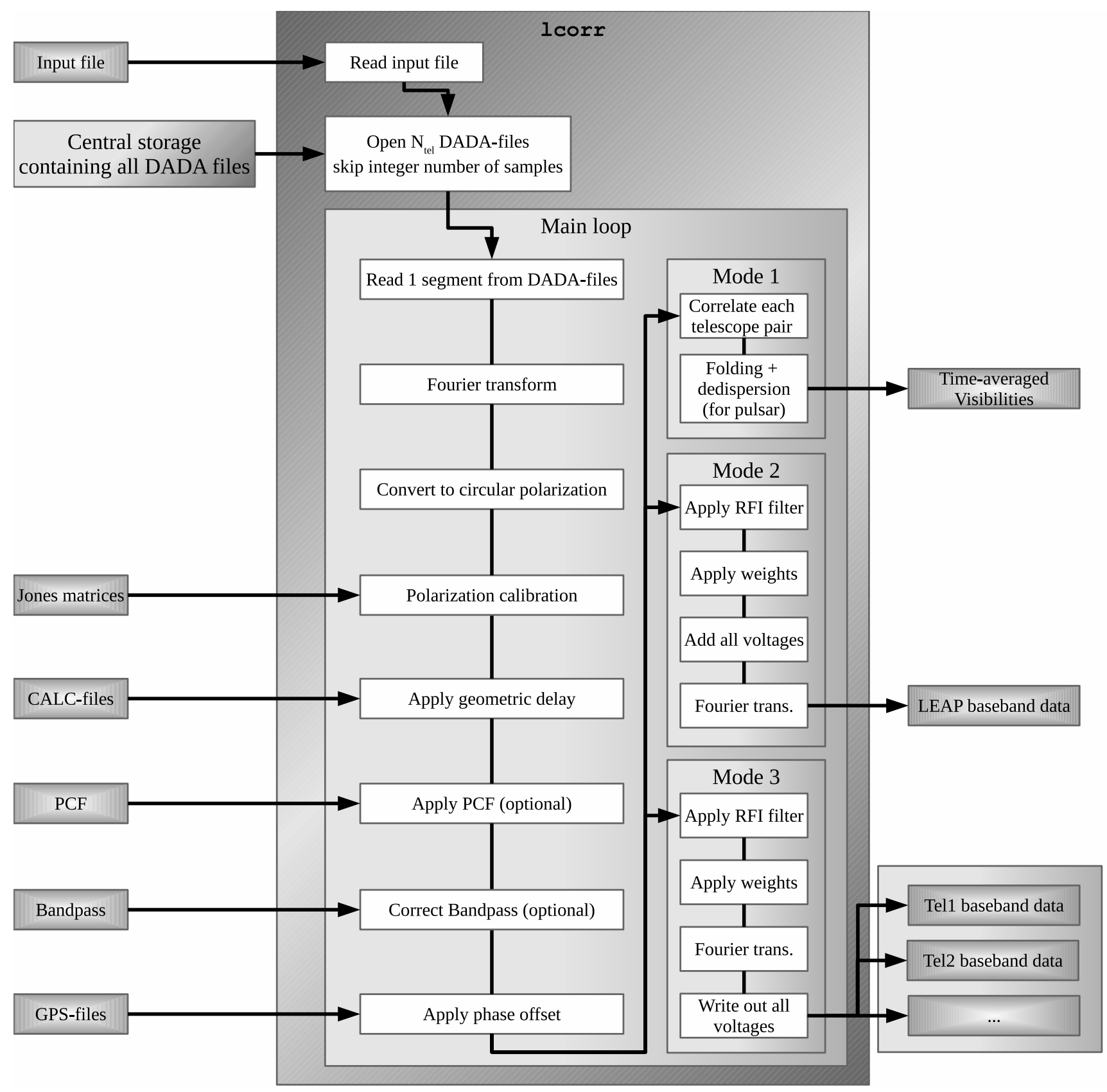

Figure 4: Flowchart of the LEAP software correlator 1corr. This chart corresponds to the 'LEAP Correlator' block in Fig. 3 but with more details. Mode 1 is the correlator mode that is used in sequence 1 and 2 from Fig. 3. Mode 2 is the addition mode that is used in sequence 3 from Fig. 3. Mode 3 is an extra mode in which the complex samples are written to disk which allows them to be added incoherently. See text for a detailed explanation of this chart. 
CALC files contain the polynomials from which the exact geometric delays are calculated for each observatory with EB as the reference point. The frequency-dependent phase offsets are stored in the PCF (see Section 4.4). After reading the GPS clockfiles, lcorr performs a linear interpolation between the measured clock offsets to estimate the clock offset corresponding to the time of the ten-second time series. It then opens the $\mathrm{N}_{\text {tel }}$ DADA files for reading, where $\mathrm{N}_{\text {tel }}$ is the number of telescopes, and skips a number of samples corresponding to the total time offset. This leaves a time offset less than a fraction of a sample. Note that by skipping these samples, the phases of the radiowaves as received by the telescopes are shifted as well. This is corrected for at a later stage (see Section 4.8).

Within the main loop, lcorr reads the content of the DADA files for each telescope in small segments, typically 100 samples5. The samples are Fourier transformed. If the polarization of the data is linear, it is converted to circular so that the polarization from all telescopes are in the same basis. This is performed using the conversion Jones matrix as described in Hamaker \& Bregman (1996). The data is then polarization calibrated by applying the Jones matrix for that telescope (see Section 4.2) and the geometric delay is applied. Optionally, the frequency-dependent phase offsets are applied (in practice, these are always applied once they have been measured). Optionally, a bandpass correction is applied (see Section 4.3). Next, the segments from all telescopes are aligned in phase. This happens by rotating each complex sample by an angle $\phi$, which aligns the signal within the samples from all telescopes in phase (see Section 4.8). lcorr can then either multiply the voltages between each pair of telescopes, resulting in the visibilities (mode 1), add the voltages together (mode 2 ), or write out the complex samples for each telescope (mode 3 ).

If the time series are from a pulsar observation, then in mode 1 the visibilities from all frequency channels are binned into a given number of phase-bins over the pulsar period. The pulsar period during the 10 -second time series is predicted using TEMPO, a program for analyzing pulsar timing data (Manchester et al., 2015) and the pulsar parameter file (i.e. the $<$ psrname $>$.par file). The binned visibilities are dedispersed incoherently by shifting the frequency channels by the appropriate integer number of phase-bins using a cyclic boundary. For a calibrator, the visibilities from each frequency channel are simply averaged over time. In mode 2, the samples are first scaled according to a weight defined in the input file. This will correct for differences in telescope gain and optimize the $\mathrm{S} / \mathrm{N}$ (see Section 4.9). The samples are then added together and scaled to a dynamic range of 8 bits before being written to disk in the DADA file format. Mode 3 is identical to mode 2 , except that the complex samples from the telescopes are not added together, but are written to disk individually in the DADA file format which allows them to be added incoherently (see Section 4.10).

\footnotetext{
${ }^{5}$ Typically 100 samples are used for the Fourier transform of 16-MHz data and 125 samples are used for the Fourier transform of 20-MHz data, producing a filterbank with $160-\mathrm{kHz}$ channels. This ensures that the channels from both the $16-\mathrm{MHz}$ data and the $20-\mathrm{MHz}$ data from the WSRT can be matched in frequency.
}

\subsection{Polarization calibration}

A crucial part of the correlator pipeline, is an accurate polarization calibration that removes the effects introduced by the telescope, receiver and instruments. For this purpose we observe a suitable polarization calibrator with all participating telescopes as part of the observation session. This is typically a bright, highly polarized pulsar such as PSR B1933+16, or PSR J1022+1001. After dedispersion, the frequency channels are averaged together so as to yield a frequency resolution of $4 \mathrm{MHz}$, which provides both sufficient sensitivity as well as sufficient frequency resolution. Then, for each $4-\mathrm{MHz}$ channel of the pulsar observation from each telescope, a polarization calibration procedure is performed which yields the $4 \times 4$ Mueller matrices. These Mueller matrices describe the distortion of polarization and are independent of the paralactic angle. From the Mueller matrices the Jones matrices can be produced, which describe the distortion of polarization for a specific paralactic angle. Since the paralactic angle changes between sources (for the alt-azimuth mounted telescopes), there is a Jones matrix for each 4-MHz channel from each telescope and for each observation. The changes in telescope angle on the sky during an observation is usually neglected.

The Jones matrices are read by lcorr. Once the complex samples from each telescope are Fourier transformed and converted to circular polarization, the samples are multiplied by the appropriate Jones matrix, which yields the polarization calibrated complex samples. This process is performed before the addition to maximize the coherency of the tied-array beam, but also because performing the polarization calibration after addition is complicated due to the extra phase rotation that is applied to the complex samples of each telescope. Bassa et al. (2016) give a description of the LEAP polarization calibration, which is a crucial step to achieve full coherence. For this reason, a more detailed description can be found in a forthcoming paper (Lee et al., in prep.).

\subsection{Bandpass correction}

The receiving power of an antenna within a band is affected by the receiver antenna and the polyphase filter. For a perfect system, this bandpass would be flat. In practice, however, the bandpass usually has a roll-off at the edges of the band and may show other features. See for example Fig. 5 which shows the bandpass from both EB and NRT. These features have a small negative effect on the addition of the signal from different telescopes as they introduce an artificial weight on each frequency channel. The LEAP software allows for a bandpass correction. This is performed by first correlating a bright calibrator that has been observed right before the pulsar observation. From the visibilities of the autocorrelations, we extract the average receiving power of each telescope for both polarizations for each frequency channel, to obtain the bandpass. These values are stored in data files, which can be read by lcorr. The option for a bandpass correction can be activated via the input file. lcorr will then divide the complex samples in each frequency channel with the corresponding average receiving power, thus normalizing the bandpass. 

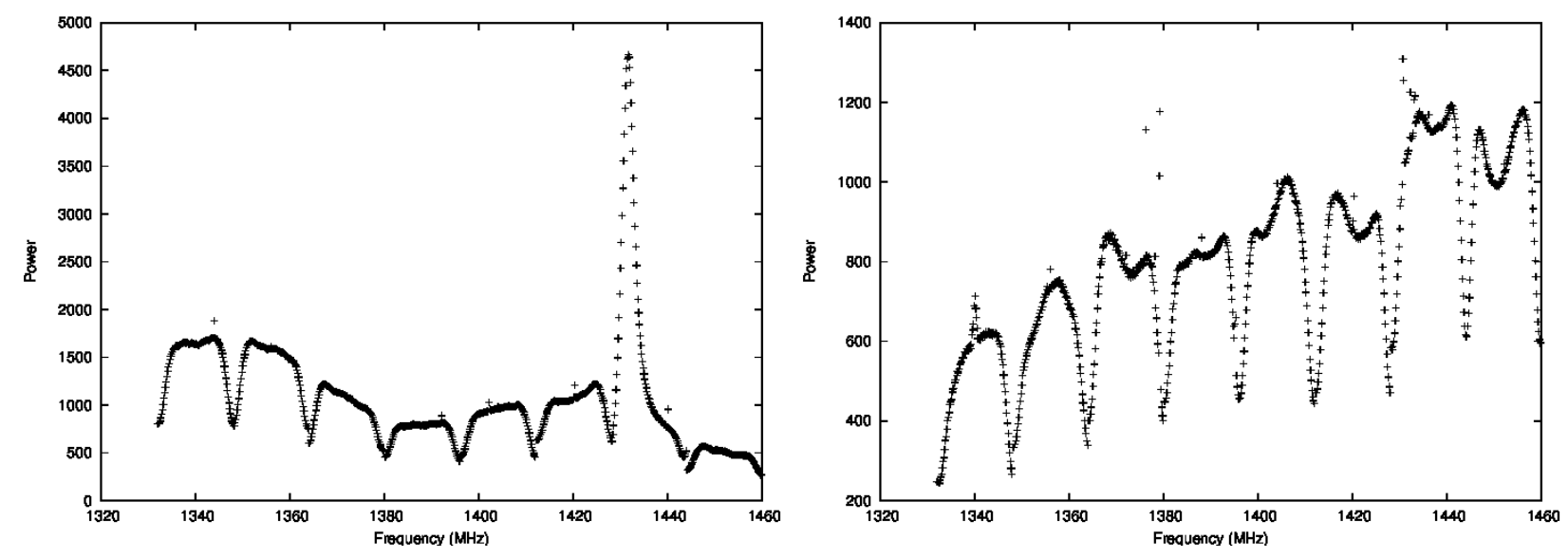

Figure 5: The bandpass of 8 16-MHz bands from EB (left) and NRT (right). After polyphase filtering, the EB bandpass shows an artificial feature around $1430 \mathrm{MHz}$ caused by a resonance in the receiver. Some channels have a clear excess of integrated power, which is caused by narrow-band RFI. Such channels will be masked by the interference mitigation (see Section 4.5).

\subsection{Frequency dependent phase correction}

The receiver antenna and the polyphase filter not only affect the power of an antenna, but also the phase of the complex sampled voltages. However, the frequency evolution of the signal's phase only becomes visible when a reference signal is present, as is the case when correlating the baseband data from 2 telescopes. Fig. 6 shows the phase of the visibilities from a calibrator between two telescopes before correcting for the frequency evolution of the signal's phase. To keep both signals in phase over the whole band, the frequency-dependent phase offset needs to be measured. This is performed by first correlating a bright calibrator that has been observed with all the telescopes. This can be the same correlation as used for the bandpass correction. From the visibilities of each pair of telescopes the phase-offset is calculated for each frequency channel and for both polarizations. These phase-offsets are stored in the PCFs, which can be read by lcorr. The option for a phase correction can be activated via the input file. lcorr will then rotate the complex sample of every visibility by the corresponding phase, removing all frequency dependence of the phases.

The combination of correcting for both the power and the phase in the bandbass is sometimes referred to as a complex bandpass correction.

\subsection{Interference mitigation}

The method for interference mitigation is described in Section 4.5 of Bassa et al. (2016). In brief, there are two techniques that are both implemented within lcorr.

The first technique involves selecting frequency channels from the Fourier transformed voltages from each telescope that contain an excess of integrated power. This technique is sensitive to narrow-band interference. Channels are selected when the power exceeds a threshold or deviates significantly from its neighbors. These channels are then masked by replacing the content with Gaussian noise with a mean and rms determined by the average of the remaining frequency channels.
The second technique implements the method of spectral kurtosis and is most sensitive to time-varying interference (see Nita \& Gary, 2010a b). In each frequency channel and at each telescope, the distribution of a time-series of 1000 samples of total power is assessed for similarity to that expected from Gaussian-distributed amplitudes. Non-Gaussian distributed amplitudes are attributed to the presence of interference. The samples from such a time-series are replaced with Gaussian noise.

\subsection{Pulsar gating}

Mode 1 of the correlator lcorr produces the visibilities from all the telescope pairs. These visibilities are then used to find the fringe-solution, which is required to align the timeseries in time and phase (see Section 4.7). The observation of a calibrator is used to find an approximate fringe-solution for the pulsar observation. Due to the temporal and spatial offset of the calibrator that solution will deviate from the required fringesolution for the pulsar observation. Also, during the course of the pulsar observation any fixed fringe-solution will become invalid due to clock drifts and the changing ionosphere and troposphere. It is thus required to calibrate on the pulsar signal itself. The processing of the pulsar timeseries is performed in several parts to allow for different fringe-solutions over the course of the observation. These parts are typically five to ten minutes long. The resulting visibility files contain the visibilities from all the telescope pairs in a given number of phase-bins, where all the phase-bins make up one pulse period. Typically, the number of phase-bins is about 30 . For each of these phasebins the average power is calculated and the pulsar profile is created. Phase-bins that have a power of more than 3 sigma above the noise-level are selected as the on-pulse. This process is called 'gating'. The visibilities of the on-pulse bins are then averaged together, which improves the $\mathrm{S} / \mathrm{N}$. The resulting visibilities are used to find a fringe-solution. 


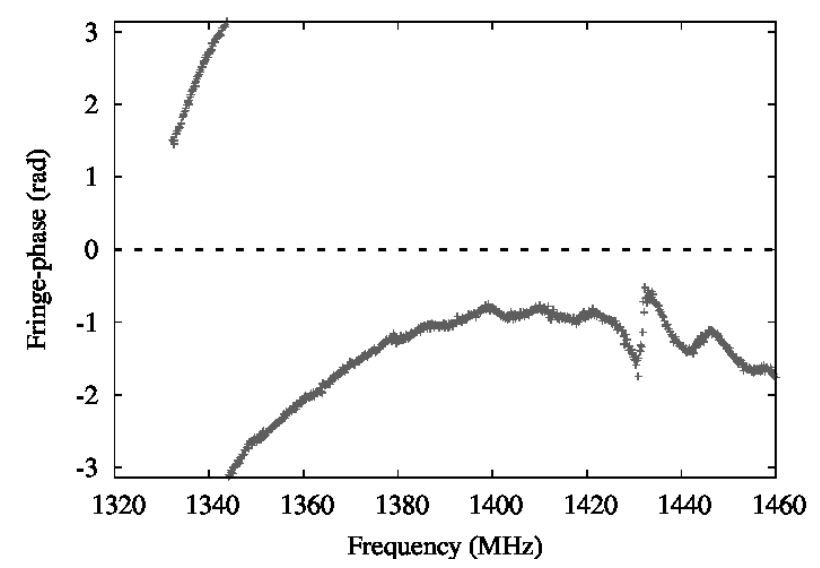

Figure 6: Phase of the visibilities from calibrator J1025+1253 between telescopes EB and NRT as a function of frequency channel before correcting for the frequency-dependent phase offset.

\subsection{Finding fringes}

The correlations are run in parts, where each part contains all the bands and all the 10-second time-series over a given timeinterval of typically five to ten minutes. After the correlations from one part are finished, all resulting visibilities are merged together to form one visibility file. For a pulsar observation, the visibilities are gated (see Section 4.6). The merged visibility file is used to find the fringe-solution. The fringe-search is performed via the C-based program fringefind, which applies the method of Schwab \& Cotton (1983) in two stages. In the first stage the visibilities are Fourier transformed and each resulting complex sample is multiplied by its complex conjugate to yield a power series. From this series, the sample with maximum is power is selected, which corresponds to a delay which is a multiple of the Nyquist sampling of the original voltages (i.e. $62.5 \mathrm{~ns}$ for the $16-\mathrm{MHz}$ bands). In the second stage this integer shift is applied to the visibilities. A least-squares algorithm is used that minimizes the difference between model phases and measured phases by solving for the fringe phase, the fringe delay and the fringe rate between each pair of telescopes. The resulting solution is restricted by the requirement of phase closure. The fits are performed independently on both lefthand-circular and right-hand-circular polarizations. The resulting fringe rates are averaged over both polarizations. Typically the initial fringe-solution is found using a calibrator. This solution is then applied to the pulsar observation, which places the fringe-delays between each pairs of telescopes already within one Nyquist sample.

\subsection{Correcting the time delays}

Before the addition, the complex samples from all telescopes are aligned in phase. This happens by rotating each complex sample by an amount $\phi[k, p, t]$, as defined in Eq.1 and obtained via the procedure described in Section 4.7. After the complex samples from each telescope are rotated by the angle $\phi$, the contributions from the pulsar (or calibrator) signal are in phase, whereas the contribution from the noise remain with a random phase-offset.

\subsection{Coherent addition of the voltages}

Once the complex samples from each pair of telescopes are in phase, they can be added with lcorr (mode 2) to form the tied array beam. As described in Section 4.4 of Bassa et al. (2016) we have to apply an appropriate weight to the baseband data from each of the telescopes, to ensure maximum $\mathrm{S} / \mathrm{N}$ of the added data. The program lcorr measures the average noise levels of both polarizations for each telescope from the baseband data, which is used to normalize all samples to an equal noise level. It then applies a weight based on the $\mathrm{S} / \mathrm{N}$ of the average intensity profiles from the individual telescopes, given by:

$$
W_{\mathrm{tel}}=\sqrt{\frac{\mathrm{S} / \mathrm{N}_{\mathrm{tel}}}{\mathrm{S} / \mathrm{N}_{\mathrm{ref}}}},
$$

where $S / N_{\text {tel }}$ is the $S / N$ of the telescope and $S / N_{\text {ref }}$ is the $S / N$ of a reference telescope. Finally, the samples are again normalized such that the resulting added samples have a proper dynamic range, yet minimum saturation when they are converted to 8 bit. This normalization factor is given by $35 / \Sigma_{i=1}^{\mathrm{N}_{\text {tel }}} W_{i}$, where 35 is the rms of the added samples and is chosen such that less than $0.3 \%$ of the samples are clipped.

Once the complex samples are properly scaled, they are added together, Fourier transformed back into the time domain and written to disk as 8-bit samples in the DADA format.

\subsection{Incoherent addition of the voltages}

The coherent addition of the pulsar signal from different telescopes requires a fringe solution which is obtained from correlating the complex sampled voltages from each telescope. However, some observations only have a very weak pulsar signal which can fail to yield a fringe solution. In that case it is still beneficial to add the samples together incoherently. This is achieved by using mode 3 of lcorr which aligns the complex samples from each telescope by applying the geometric delays, the clock offset, the instrument specific delays and the fringe solution from the calibrator. This aligns the samples from 
the different telescopes to about $10 \mathrm{~ns}$, depending on the conditions of the ionosphere and the calibrator angular separation from the pulsar. The same weights as described in Section 4.9 are applied, the frequency channels from each telescope are Fourier transformed back to the time domain and are written to disk as 8-bit samples. Each time series is then dedispersed using DSPSR. The resulting archive files are added with PSRADD, which is part of the PSRCHIVE software (Hotan et al., 2004).

\section{Summary}

As part of LEAP, we have developed a software correlator and beamformer that allows the pulsar observations from the telescopes from the EPTA to be added coherently, thus significantly improving their effective area to that of a steerable 195-m circular dish. Another important improvement is the accurate polarization calibration that is performed before the individual time-series are added together. In this paper we have presented a detailed overview of the software pipeline and the techniques that are used. In particular it describes the steps that are required to calibrate, correlate and add the complex sampled time series from the EPTA telescopes. It also gives a detailed outline of the C-based program lcorr, which lies at the heart of the correlator and beamformer. The majority of the software and techniques can be applied to other telescopes as well. All software is available for download from http://www.epta.eu.org/aom.html.

\section{Acknowledgements}

The European Pulsar Timing Array (EPTA) is a collaboration of European institutes to work towards the direct detection of low-frequency GWs and to implement the Large European Array for Pulsars (LEAP). The authors acknowledge the support of the colleagues in the EPTA. We also like to thank our colleagues A. Deller, M. Kettenis and Z. Paragi for sharing their knowledge of VLBI and an anonymous referee for their comments. The work reported in this paper has been funded by the ERC Advanced Grant "LEAP", Grant Agreement Number 227947 (PI M. Kramer). Pulsar astronomy at the University of Manchester and access to the Lovell telescope are funded through a consolidated grant from STFC. RS acknowledges support from the European Research Council under the European Union's Seventh Framework Programme (FP/2007-2013) / ERC Grant Agreement n. 617199.

\section{References}

Abbott B. P., et al., 2016, Physical Review Letters, 116, 061102

Bassa C. G., et al., 2016, MNRAS, 456, 2196

Deller A. T., Tingay S. J., Bailes M., West C., 2007, PASP, 119, 318

Demorest P. B., et al., 2013, ApJ, 762, 94

Desvignes G., et al., 2016, MNRAS, 458, 3341

Detweiler S., 1979, ApJ, 234, 1100

Haehnelt M. G., 1994, MNRAS, 269, 199

Hamaker J. P., Bregman J. D., 1996, A\&AS, 117, 161

Hellings R. W., Downs G. S., 1983, ApJ, 265, L39

Hotan A. W., van Straten W., Manchester R. N., 2004, Publ. Astron. Soc. Australia, 21, 302
Jaffe A. H., Backer D. C., 2003, ApJ, 583, 616

Karuppusamy R., 2011, in Burgay M., D’Amico N., Esposito P., Pellizzoni A., Possenti A., eds, American Institute of Physics Conference Series Vol. 1357, American Institute of Physics Conference Series. pp 89-90

Karuppusamy R., Stappers B., van Straten W., 2008, Publ. Astron. Soc. Australia, 120, 191

Keimpema A., et al., 2015, Experimental Astronomy, 39, 259

Kramer M., Stappers B., 2010, in ISKAF2010 Science Meeting. p. 34

Lee K. J., Wex N., Kramer M., Stappers B. W., Bassa C. G., Janssen G. H., Karuppusamy R., Smits R., 2011, MNRAS, 414, 3251

Lentati L., et al., 2015, MNRAS, 453, 2576

Lommen A. N., 2012, Journal of Physics Conference Series, 363, 012029

Manchester R. N., et al., 2013, Proc. Astr. Soc. Aust., 30, e017

Manchester R., et al., 2015, Tempo: Pulsar timing data analysis, Astrophysics Source Code Library (ascl:1509.002)

Nita G. M., Gary D. E., 2010a, PASP, 122, 595

Nita G. M., Gary D. E., 2010b, MNRAS, 406, L60

Ryan J. W., Vandenberg N. R., 1980, in Bulletin of the American Astronomical Society. p. 457

Sanidas S. A., Battye R. A., Stappers B. W., 2012, Phys. Rev. D, 85, 122003

Schwab F. R., Cotton W. D., 1983, AJ, 88, 688

Sesana A., Vecchio A., Colacino C. N., 2008, MNRAS, 390, 192

Shannon R. M., et al., 2015, Science, 349, 1522

Taylor J. H., 1992, Philosophical Transactions of the Royal Society of London Series A, 341, 117

Taylor J. H., Weisberg J. M., 1982, ApJ, 253, 908

The NANOGrav Collaboration et al., 2015, ApJ, 813, 65

van Straten W., Bailes M., 2011, Publ. Astron. Soc. Australia, 28, 1

van Straten W., Demorest P., Oslowski S., 2012, Astronomical Research and Technology, 9, 237 